\title{
Selective dissociation between LSD1 and GFI1B by a LSD1 inhibitor NCD38 induces the activation of ERG super-enhancer in erythroleukemia cells
}

\author{
Ryusuke Yamamoto ${ }^{1}$, Masahiro Kawahara ${ }^{1,2}$, Shinji Ito ${ }^{3}$, Junko Satoh ${ }^{3}$, Goichi \\ Tatsumi ${ }^{1}$, Masakatsu Hishizawa ${ }^{1}$, Takayoshi Suzuki ${ }^{4,5}$, and Akira Andoh ${ }^{2}$ \\ ${ }^{1}$ Department of Hematology and Oncology, Graduate School of Medicine, Kyoto University, Kyoto, Kyoto, Japan \\ ${ }^{2}$ Department of Medicine, Shiga University of Medical Science, Otsu, Shiga, Japan \\ ${ }^{3}$ Medical Research Support Center, Graduate School of Medicine, Kyoto University, Kyoto, Kyoto, Japan \\ ${ }^{4}$ Department of Chemistry, Graduate School of Medical Science, Kyoto Prefectural University of Medicine, Kyoto, Kyoto, Japan \\ ${ }^{5}$ CREST, Japan Science and Technology Agency (JST), Kawaguchi, Saitama, Japan \\ Correspondence to: Masahiro Kawahara, email: mkawahar@belle.shiga-med.ac.jp
}

Keywords: LSD I; super-enhancer; GFIlB; RUNXI; leukemia

Received: September 05, $2017 \quad$ Accepted: February 24, $2018 \quad$ Published: April 20, 2018

Copyright: Yamamoto et al. This is an open-access article distributed under the terms of the Creative Commons Attribution License 3.0 (CC BY 3.0), which permits unrestricted use, distribution, and reproduction in any medium, provided the original author and source are credited.

\section{ABSTRACT}

Lysine-specific demethylase 1 (LSD1) is a histone modifier for transcriptional repression involved in the regulation of hematopoiesis. We previously reported that a LSD1 inhibitor NCD38 induces transdifferentiation from erythroid lineage to granulomonocytic lineage and exerts anti-leukemia effect through de-repression of the specific super-enhancers of hematopoietic regulators including ERG in a human erythroleukemia cell line, HEL. However, the mechanistic basis for this specificity of NCD38 has remained unclear. Herein, we report major partners associated with LSD1 and clarify the mechanism in HEL cells. Proteome analysis identified 54 candidate proteins associated with LSD1, including several transcription factors such as GFI1B and RUNX1 as well as BRAF-histone deacetylase complex (BHC) components such as COREST, HDAC1, and HDAC2. NCD38 selectively disrupted the interaction of LSD1 with GFI1B but not with RUNX1, CoREST, HDAC1 and HDAC2. Erg was downregulated in murine erythroid progenitors with prominent upregulation of Gfi1b. NCD38 induced ERG and attenuated an erythroid marker CD235a in HEL while this attenuation was mimicked by the lentiviral overexpression of ERG. The ERG super-enhancer contained the conserved binding motif of GFI1B and was actually occupied by GFI1B. NCD38 dissociated LSD1 and CoREST but not GFI1B from the ERG super-enhancer. Collectively, the selective separation of LSD1 from GFI1B by NCD38 restores the ERG super-enhancer activation and consequently upregulates ERG expression, inducing the transdifferentiation linked to the anti-leukemia effect.

\section{INTRODUCTION}

Transcription factors (TFs) play essential roles in the regulation of normal hematopoiesis [1]. Abnormalities and dysregulation of TFs are major causes of leukemogenesis
[2-4]. TFs cooperatively or antagonistically regulate each other within transcriptional networks [5], and this regulation is controlled via the activation of enhancers near or inside the gene bodies of TFs [6]. As enhancers contain multiple binding motifs of TFs, they are variously 
activated depending on TFs recruitment. In particular, super-enhancers (SEs) which are concatenated enhancers confer higher transcriptional activity than typical enhancers [7]. Activated SEs are found near the genes that define cell identity in embryonic stem cells and oncogenes in tumor cells, indicating that the precise control of SEs is essential in normal cell differentiation and dysregulation of SEs links to tumor pathogenesis [8]. SEs are occupied not only by TFs but also by mediators, chromatin regulators and transcription apparatus, and activated in a lineage specific manner [7]. On the other hand, the proper shut-down of SEs that are no longer required during the process of differentiation is also essential to secure the lineage specificity, however, less is known about the detailed mechanism.

Lysine-specific demethylase 1 (LSD1, also known as KDM1A, BHC110, AOF2, or KIAA0601), which was identified as a histone demethylase and a transcriptional corepressor [9], possesses a flavin containing amine oxidase that catalyzes mono- and di-methylated histone 3 lysine 4 (H3K4me1 and H3K4me2). LSD1 also interacts with RE1 silencing transcription factor corepressor (CoREST, also known as RCOR1) and histone deacetylase 1 and 2 (HDAC1 and HDAC2, respectively) [10, 11]. LSD1 is therefore involved in deacetylation of acetylated histone 3 lysine 27 (H3K27ac). Histone modifications are a crucial manner to regulate gene transcriptions [12]. In particular, as $\mathrm{H} 3 \mathrm{~K} 4 \mathrm{me} 1$ and $\mathrm{H} 3 \mathrm{~K} 27 \mathrm{ac}$ are general markers of poised or activated enhancers [13], LSD1 is supposed to function as a repressor of enhancers. In fact, loss of Lsd 1 causes pancytopenia and is associated with increased levels of H3K27ac on the enhancers of Lsd1 target genes in murine hematopoietic-lineage cells [14].

We recently reported that a novel LSD1 inhibitor, NCD38, can induce myeloid differentiation in human erythroleukemia (HEL) cells and activate approximately 500 SEs [15]. According to the rank order SE (ROSE) analysis, the SE of Ets related gene (ERG), one of the key TFs for hematopoiesis [5], was at the highest rank among them. These data indicate that LSD1 is involved in blood cell differentiation through repressing the SEs of the key hematopoietic TFs, however, it has remained unclear how LSD1 preferentially regulates such SEs. In this study, we attempt to identify binding partners for LSD1 in HEL cells, and to clarify the mechanism that NCD38 can selectively de-repress particular SEs with these partners, focusing on the $E R G$-SE.

\section{RESULTS}

\section{Identification of LSD1 partners in HEL cells}

To identify proteins that are associated with LSD1 in HEL cells, we first purified the whole-cell lysate by co-immunoprecipitation (co-IP) with anti-LSD1 antibody (LSD1-IP). The LSD1-IP lysate showed not only a protein band corresponding to the size of LSD1 but also several protein bands which were not detected in the control IgGIP lysate (Figure 1A). Liquid chromatography coupled with tandem mass spectrometry (LC-MS/MS) analyses successfully revealed a number of peptides in the LSD1IP sample (Figure 1B). Finally, as LSD1 partners in HEL cells, we identified 54 proteins of which more than one peptide were detected with at least $95 \%$ confidence in the LSD1-IP sample but not in the control IgG-IP sample and which had reliable score calculated by the Pro Group algorithm [Unused ProtScore $\geq 2$ ] (Table 1). They included components of BRAF-histone deacetylase complex (BHC) such as CoREST (also known as RCOR1), HDAC1, HDAC2, GSE1, HMG20A and HMG20B which were previously reported to form the LSD1-containing complex $[11,16]$. In addition, key hematopoietic TFs such as RUNX1 and GFI1B were listed in the LSD1 partner list (Table 1).

The LSD1-CoREST-HDAC1/2 complex was previously identified in various types of cells including neural cells, HEK293T cells, Hela cells, and blood cells $[10,11,16,17]$, and was reported to interact with GFI1B in murine blood cells [17]. To confirm the proper formation of the LSD1-CoREST-HDAC1/2 complex and the association of the complex with GFI1B and RUNX1 in HEL cells, we performed co-IP followed by western blotting (co-IP-WB). The formation of the LSD1CoREST-HDAC1/2 complex was validated by co-IP-WB with anti-LSD1 antibody (Figure 1C). The interaction of the LSD1-CoREST-HDAC1/2 complex with GFI1B was also validated by co-IP-WB with anti-GFI1B antibody (Figure 1D). Furthermore, co-IP-WB with anti-RUNX1 antibody showed that RUNX1 interacts with the LSD1CoREST-HDAC1/2 complex (Figure 1E). These data suggest that GFI1B and RUNX1 form a complex with LSD1, CoREST and HDAC 1/2 in HEL cells.

\section{Selective dissociation of GFI1B from the LSD1 complex by NCD38}

We previously reported that NCD38 exerts anti-leukemic effect against HEL cells through transdifferentiation from erythroid lineage to granulomonocytic lineage [15]. The other recent study also reported that another LSD1 inhibitor T-3775440 can cause the similar effect and disrupt the interaction between LSD1 and GFI1B in HEL cells [18]. Thus, we comprehensively investigated whether the interaction of LSD1 with the identified partners is altered by NCD38 treatment in HEL cells. The relative abundances of LSD1-interacting proteins in the presence or absence of NCD38 were estimated by label-free quantification (Supplementary Table 1). There were no key hematopoietic regulators which gained association with LSD1 in the presence of NCD38 by more than 1.5-folds. In contrast, the binding rate of GFI1B to LSD1 was 
Table 1: LSD1 partner candidates identified by LC-MS/MS in HEL cells

\begin{tabular}{|c|c|c|c|c|}
\hline Rank & Symbol Name & UniProtKB number & Unused ProtScore & Peptides (95\%) \\
\hline 1 & GSE1 & Q14687 & 101.90 & 55 \\
\hline 2 & KDM1A (LSD1) & O60341 & 89.31 & 62 \\
\hline 3 & HSPD1 & P10809 & 71.24 & 48 \\
\hline 4 & RCOR1 (CoREST) & Q9UKL0 & 44.71 & 28 \\
\hline 5 & HDAC2 & Q92769 & 31.70 & 23 \\
\hline 6 & HNRNPUL1 & Q9BUJ2 & 21.59 & 11 \\
\hline 7 & THRAP3 & Q9Y2W1 & 20.07 & 10 \\
\hline 8 & HMG20A & Q9NP66 & 19.73 & 11 \\
\hline 9 & SMARCA5 & O60264 & 19.05 & 10 \\
\hline 10 & RCOR3 & Q9P2K3 & 18.08 & 18 \\
\hline 11 & MYO1G & B0I1T2 & 16.31 & 9 \\
\hline 12 & BCLAF1 & Q9NYF8 & 15.64 & 8 \\
\hline 13 & HDAC1 & Q13547 & 15.01 & 21 \\
\hline 14 & RPS2 & P15880 & 13.88 & 8 \\
\hline 15 & THBS1 & P07996 & 13.77 & 7 \\
\hline 16 & HMG20B & Q9P0W2 & 13.44 & 7 \\
\hline 17 & SUPT16H & Q9Y5B9 & 12.01 & 6 \\
\hline 18 & UBTF & P17480 & 9.65 & 6 \\
\hline 19 & ERH & P84090 & 9.09 & 5 \\
\hline 20 & RPS4X & P62701 & 7.90 & 4 \\
\hline 21 & RPS13 & P62277 & 6.18 & 3 \\
\hline 22 & RPL30 & P62888 & 6.00 & 3 \\
\hline 23 & RUNX1 & Q01196 & 5.96 & 3 \\
\hline 24 & NOP58 & Q9Y2X3 & 5.78 & 4 \\
\hline 25 & TUFM & P49411 & 5.54 & 3 \\
\hline 26 & DKC1 & O60832 & 5.27 & 4 \\
\hline 27 & HRNR & Q86YZ3 & 4.76 & 4 \\
\hline 28 & $\mathrm{MDC} 1$ & Q14676 & 4.47 & 2 \\
\hline 29 & KHDRBS1 & Q07666 & 4.36 & 2 \\
\hline 30 & SNU13 & P55769 & 4.14 & 2 \\
\hline 31 & TFAM & Q00059 & 4.01 & 2 \\
\hline 32 & HNRNPD & Q14103 & 4.00 & 2 \\
\hline 33 & PPP1CA & P62136 & 4.00 & 2 \\
\hline 34 & ILF2 & Q12905 & 4.00 & 2 \\
\hline 35 & RPA3 & P35244 & 4.00 & 2 \\
\hline 36 & FABP5 & Q01469 & 3.89 & 2 \\
\hline 37 & RPLP0 & P05388 & 3.64 & 2 \\
\hline 38 & MYO1C & O00159 & 3.63 & 2 \\
\hline 39 & RPL28 & P46779 & 3.61 & 3 \\
\hline 40 & RALY & Q9UKM9 & 3.54 & 2 \\
\hline 41 & RPL36 & Q9Y3U8 & 3.48 & 2 \\
\hline 42 & SAFB2 & Q14151 & 3.34 & 7 \\
\hline 43 & RPL32 & P62910 & 3.24 & 2 \\
\hline
\end{tabular}




\begin{tabular}{lcccc}
44 & ARPC4 & P59998 & 3.09 & 2 \\
45 & SAP18 & O00422 & 3.05 & 2 \\
46 & GFI1B & Q5VTD9 & 2.86 & 2 \\
47 & RPA1 & P27694 & 2.67 & 2 \\
48 & PPP1R9B & Q96SB3 & 2.61 & 2 \\
49 & DDX21 & Q9NR30 & 2.59 & 2 \\
50 & DSP & P15924 & 2.47 & 2 \\
51 & RPS24 & P62847 & 2.45 & 2 \\
52 & PRDX1 & Q06830 & 2.43 & 2 \\
53 & ILF3 & Q12906 & 2.40 & 10 \\
54 & H3F3A & P84243 & 2.00 & 2 \\
55 & PHOX2B & Q99453 & 2.00 & \\
\hline
\end{tabular}

Only proteins with Peptides $(95 \%) \geq 2$ and Unused ProtScore $\geq 2$ are shown.

Unused ProtScore for a particular protein was calculated from the sum of all peptide evidence for that specific protein by the Pro Group algorithm.

Peptides (95\%) means the number of distinct peptides having at least $95 \%$ confidence.

markedly reduced to $27 \%$ after the NCD38 treatment. Of note, GFI1B showed the highest reduction in the binding rate to LSD1 while no other proteins exhibited such high reduction rate (Figure 2A). In fact, the binding rate of RUNX1 to LSD1 remained at a decrease to $69 \%$ and the binding of CoREST, HDAC1, and HDAC2 to LSD1 were retained irrespective of the NCD38 treatment. To validate the binding alterations of these proteins estimated by the mass spectrometry, we next performed co-IP-WB in the presence or absence of NCD38. Co-IP-WB with antiLSD1 antibody revealed no impairment of the LSD1CoREST-HDAC1/2 complex by NCD38 (Figure 2B). In clear contrast, co-IP-WB with anti-GFI1B antibody showed that NCD38 almost completely impaired the interaction of GFI1B with LSD1, CoREST, HDAC1 and HDAC2, supporting the data obtained from the mass spectrometry (Figure 2C). Co-IP-WB with anti-RUNX1 antibody showed that the interaction of RUNX1 with LSD1, CoREST, HDAC1, and HDAC2 was less impaired by NCD38 (Figure 2D). Altogether, these data suggest that a pharmacological action of NCD38 is a selective disruption of the interaction between GFI1B and the LSD1-CoREST-HDAC1/2 complex.

\section{Inverse correlation between $E R G$ and $G F I 1 B$ transcripts}

$E R G$ was previously identified as one of the LSD1 target genes in HEL and other cell lines of acute myeloid leukemia and myelodysplastic syndromes [15]. ERG and GFI1B are known to be required for normal hematopoiesis $[19,20]$ and for erythroid maturation [21] respectively. Thus, we investigated the correlation between Erg and Gfilb transcripts in developmental stages of murine hematopoiesis [22] (Supplementary Figure 1). The Erg transcript level was high in short-term hematopoietic stem cells (ST-HSCs) and multipotent progenitors (MPPs) but relatively decreased in common myeloid progenitors (CMPs) and was much lower in megakaryocyte-erythroid progenitors (MEPs) which are in the primitive stage of erythroid lineage (Figure 3A). In contrast, the Gfilb transcript level relatively increased in CMPs and was much higher in MEPs in accordance with a previous report [23]. Furthermore, the $E R G$ transcript was hardly detected in the basal state while that was induced after the NCD38 treatment in HEL cells (Figure 3B). These data suggest that the expression of ERG and GFI1B seems to be inversely correlated in hematopoiesis and give rise to the possibility that ERG might be suppressed by GFI1B in coordination with LSD1 in immature erythroid-lineage cells.

\section{Downregulation of an erythroid marker CD235a by ERG overexpression}

We next investigated whether upregulation of ERG could be responsible for the transdifferentiation of HEL cells induced by NCD38. Using the lentiviral transduction system, we successfully overexpressed ERG at the protein level comparable to that induced by NCD38 (Figure 4A, Supplementary Figure 2). NCD38 downregulated an erythroid lineage marker, CD235a (Figure 4B), and upregulated a myeloid lineage marker, CD11b (Figure 4C). On the other hand, lentiviral ERG overexpression caused comparable downregulation of CD235a (Figure 4B) but no change of CD11b (Figure 4C). These results clearly demonstrate that ERG overexpression attenuates the erythroid-lineage phenotype of HEL cells, suggesting that upregulation of ERG seems to contribute at least in part to the transdifferentiation by NCD38. 


\section{Conservation of the GFI1B motif in the $E R G$-SE}

In our previous study, chromatin immunoprecipitation coupled with massively parallel sequencing (ChIP-seq) analysis for $\mathrm{H} 3 \mathrm{~K} 27 \mathrm{ac}$ revealed that NCD38 activates approximately 500 SEs, among which the ERG-SE is one of the highest ranked SEs according to the ROSE analysis [15]. Therefore, the pronounced increase of the ERG transcript by NCD38 is assumed to be caused by cancellation of suppression of the $E R G-\mathrm{SE}$ by LSD1. Because the ERG-SE area is highly conserved across vertebrates (Figure 5A), we surveyed the presence of LSD1 in the murine genomic region corresponding to the human ERG-SE using publicly available ChIP-seq data [24-26]. Analysis of ChIP-seq data from murine erythroleukemia (MEL) cells showed that both LSD1 and CoREST occupy the conserved SE region at the Erg gene. (Figure 5B). Moreover, the TF motif analysis revealed that the human $E R G$-SE contains the binding motifs of GFI1B and RUNX1, and that the binding motif of GFI1B but not RUNX1 is conserved across vertebrates (Figure 5C). These in silico findings suggest the possibility that LSD1 could suppress the $E R G$-SE by forming the complex with GFI1B in erythroleukemia cells.

\section{Reactivation of the $E R G$-SE by separating LSD1 and CoREST from GFI1B by NCD38}

To clarify the above possibility, we first investigated whether GFI1B specifically occupies the ERG-SE in

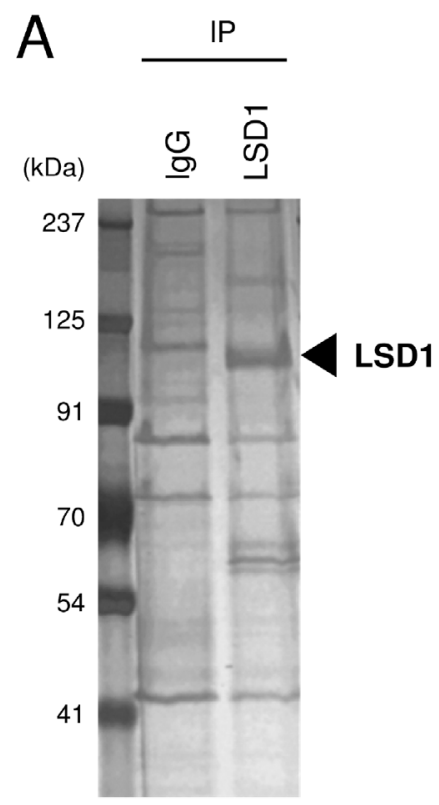

B

GFI1B

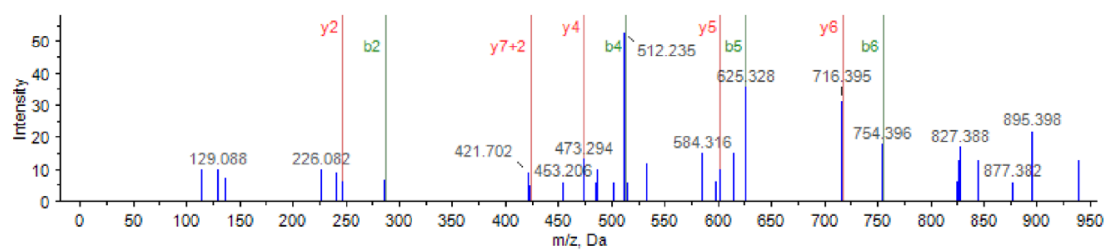

RUNX1

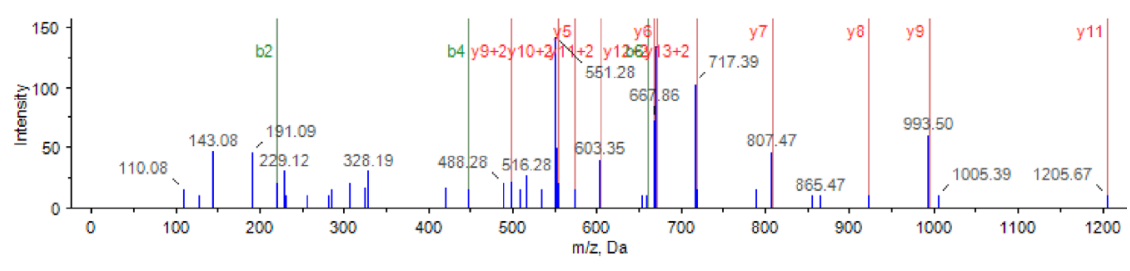

D

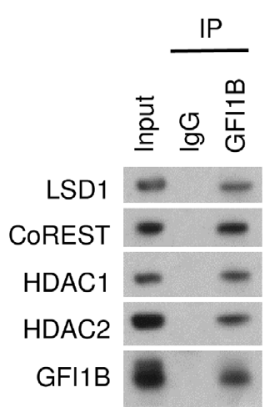

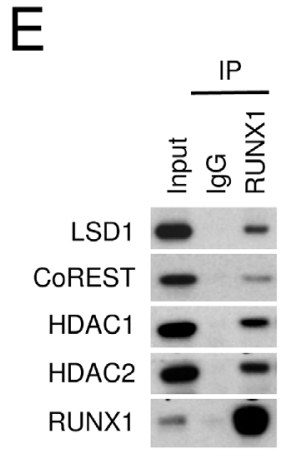

Figure 1: Interaction of LSD1 with GFI1B and RUNX1 in HEL cells. (A) Silver staining of the LSD1-IP lysate. The IP lysate with normal IgG was used as a negative control. The left lane displays size markers. The arrow indicates the protein band corresponding to the size of LSD1. (B) Representative spectra of MS/MS show the identified sequence of the peptides derived from GFI1B and RUNX1 in the LSD1-IP lysate. (C-E) Co-IP-WB with anti-LSD1 (C), anti-GFI1B (D), or anti-RUNX1 antibody (E) in HEL cells. Normal IgG was used as a negative control. Indicated antibodies were used to detect target proteins. ACTIN was used as a negative control unbound to LSD1. Experiments in $(\mathrm{C}-\mathrm{E})$ were performed independently at least three times and the representative data are shown. 
HEL cells. Chromatin-immunoprecipitation followed by quantitative PCR (ChIP-qPCR) analysis for GFI1B and RUNX1 was performed at highly conserved noncoding areas including upstream, promotor, 3'UTR, and $\mathrm{SE}$ regions (indicated in Figure 5A). As a result, GFI1B highly condensed at the SE region compared to other regions, while RUNX1 mainly condensed at the upstream region (Figure 6A). Furthermore, ChIP-qPCR analysis for $\mathrm{H} 3 \mathrm{~K} 27 \mathrm{ac}$ revealed that the level of $\mathrm{H} 3 \mathrm{~K} 27 \mathrm{ac}$ was elevated only at the SE region after the NCD38 treatment (Figure $6 \mathrm{~B})$. These results indicate that GFI1B but not RUNX1 specifically binds to the $E R G$-SE and could be involved in suppression of the $E R G$-SE. We next investigated how NCD38 alters the binding status of GFI1B, LSD1, and CoREST to the ERG-SE. ChIP-qPCR analyses at the SE region showed that the occupancy level of GFI1B was not changed (Figure 6C) but that of LSD1 and CoREST was reduced by NCD38 (Figure 6D, 6E). These results indicate

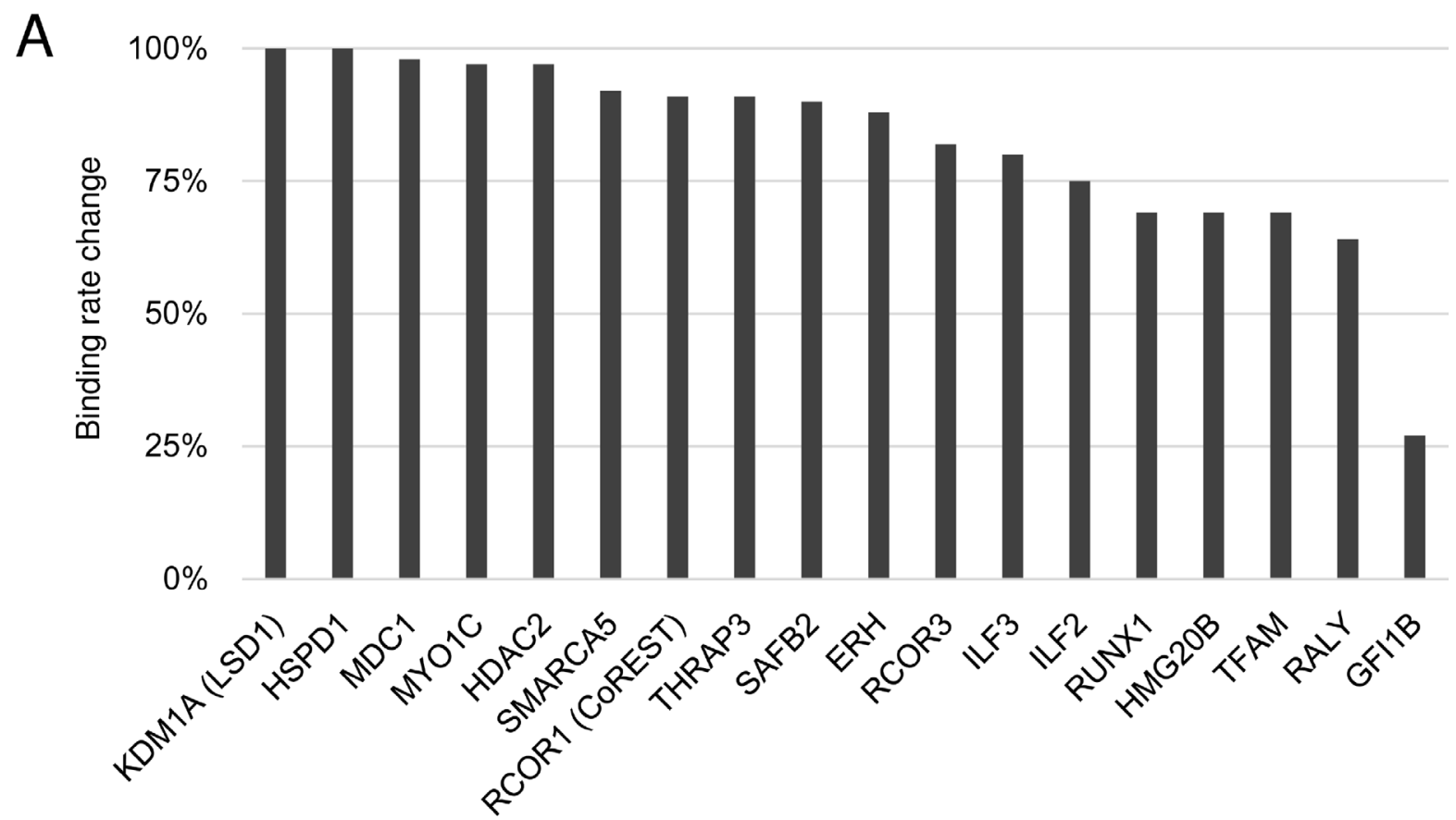

B
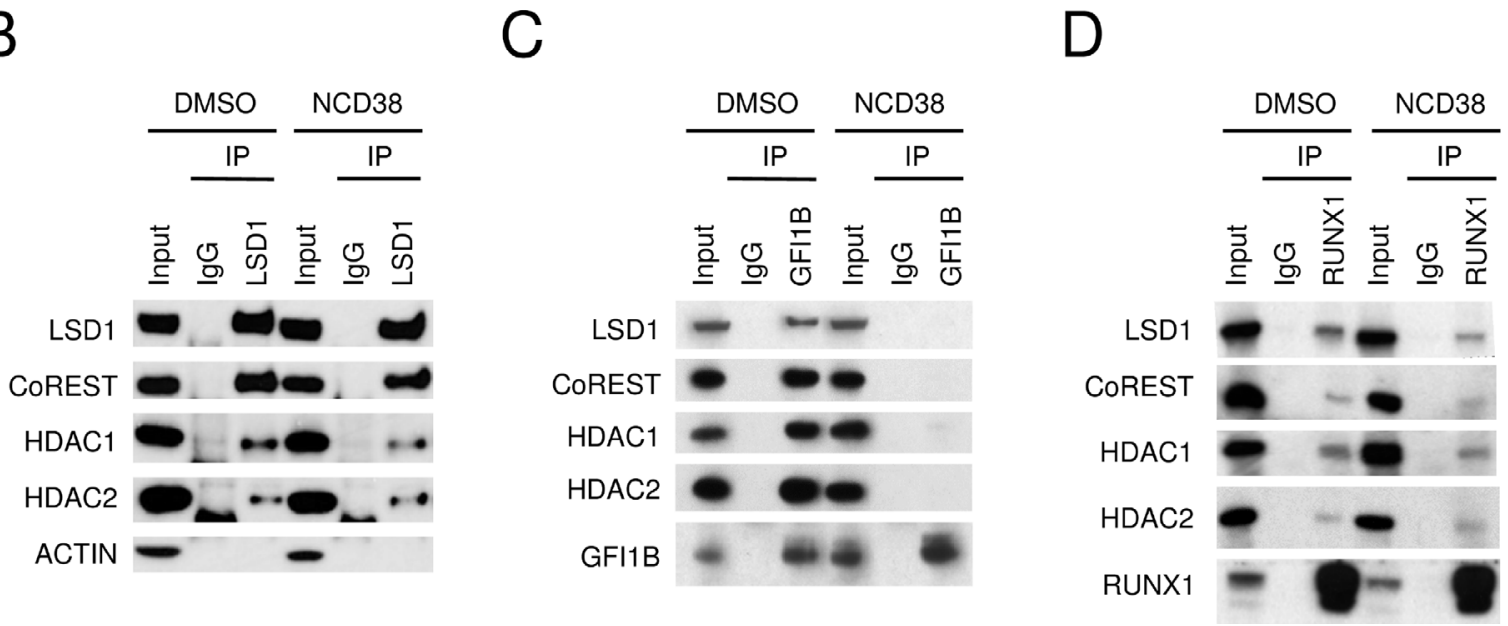

Figure 2: Selective impairment of the interaction between LSD1 and GFI1B by NCD38. (A) Binding rate change of LSD1 partners after the NCD38 treatment. The binding rate change was calculated as the normalized abundances in NCD38-treated cells divided by those in DMSO-treated cells. Values normalized to the binding rate change of LSD1 are shown. (B-D) Co-IP-WB with anti-LSD1 (B), anti-GFI1B (C), or anti-RUNX1 antibody (D) in HEL cells treated with DMSO or NCD38 for 24 hours. Normal IgG was used as a negative control. Indicated antibodies were used to detect target proteins. ACTIN was used as a negative control unbound to LSD1. Experiments in (B-D) were performed independently at least three times and the representative data are shown. 
that the binding of GFI1B to the $E R G$-SE is retained while LSD 1 and CoREST are dissociated from the ERG-SE by NCD38. Given the selective disruption of the interaction between GFI1B and the LSD1-CoREST-HDAC1/2 complex by NCD38, these data collectively suggest that GFI1B represses the ERG-SE through recruiting the complex and that NCD38 restores the activity of the $E R G$ SE by separating the complex from GFI1B (Figure 6F).

\section{DISCUSSION}

The comprehensive proteome analysis identified GFI1B and RUNX1 as major LSD1 partners that can recruit LSD1 on specific genomic regions in HEL cells. Other key hematopoietic TFs were not listed up as significant LSD1 partners at least in our result. GFI1B has been reported to interact with the LSD1-CoRESTHDAC1/2 complex via the SNAG repression domain and suppress myeloid differentiation [17]. RUNX1 has been reported to interact with LSD1 and CoREST to repress hematopoietic genes in differentiated MEL cells [27]. Therefore, GFI1B and RUNX1 presumably play crucial roles in erythroid-lineage differentiation or maintenance by utilizing LSD1. However, it is unclear how LSD1 selectively interacts with these two TFs in HEL cells, even though another study reported that LSD1 is associated with the T cell leukemia oncoprotein (TAL1/SCL) [28], which functions essentially in the early development of hematopoiesis [29]. This selectivity of LSD1 to GFI1B and RUNX1 is required to be investigated in the future.

Several LSD1 inhibitors including NCD38 are promising as anti-leukemic agents while the detailed mechanisms are less known [15, 30, 31]. Our previous report demonstrated that NCD38 induces transdifferentiation from erythroid lineage to granulomonocytic lineage with de-repression of approximately $500 \mathrm{SEs}$ in HEL cells [15]. Therefore, in the current study, we attempted to comprehensively understand the relationship between LSD1 and TFs on SEs using the ERG-SE as an example. The comprehensive proteome comparison between the presence and absence of NCD38 revealed that NCD38 selectively disrupts the interaction between LSD1 and GFI1B. Furthermore, the ChIP-qPCR analyses clearly showed that GFI1B specifically occupies the ERG-SE and that LSD1 and CoREST but not GFI1B are dissociated from the $E R G$ SE by NCD38. These data indicate that the selective dissociation of LSD1 from GFI1B on the ERG-SE by NCD38 restores the ERG-SE activation inducing ERG expression. In addition, the lentiviral ERG overexpression fulfilled a part of the transdifferentiation by NCD38. Collectively, this selective dissociation could contribute, at
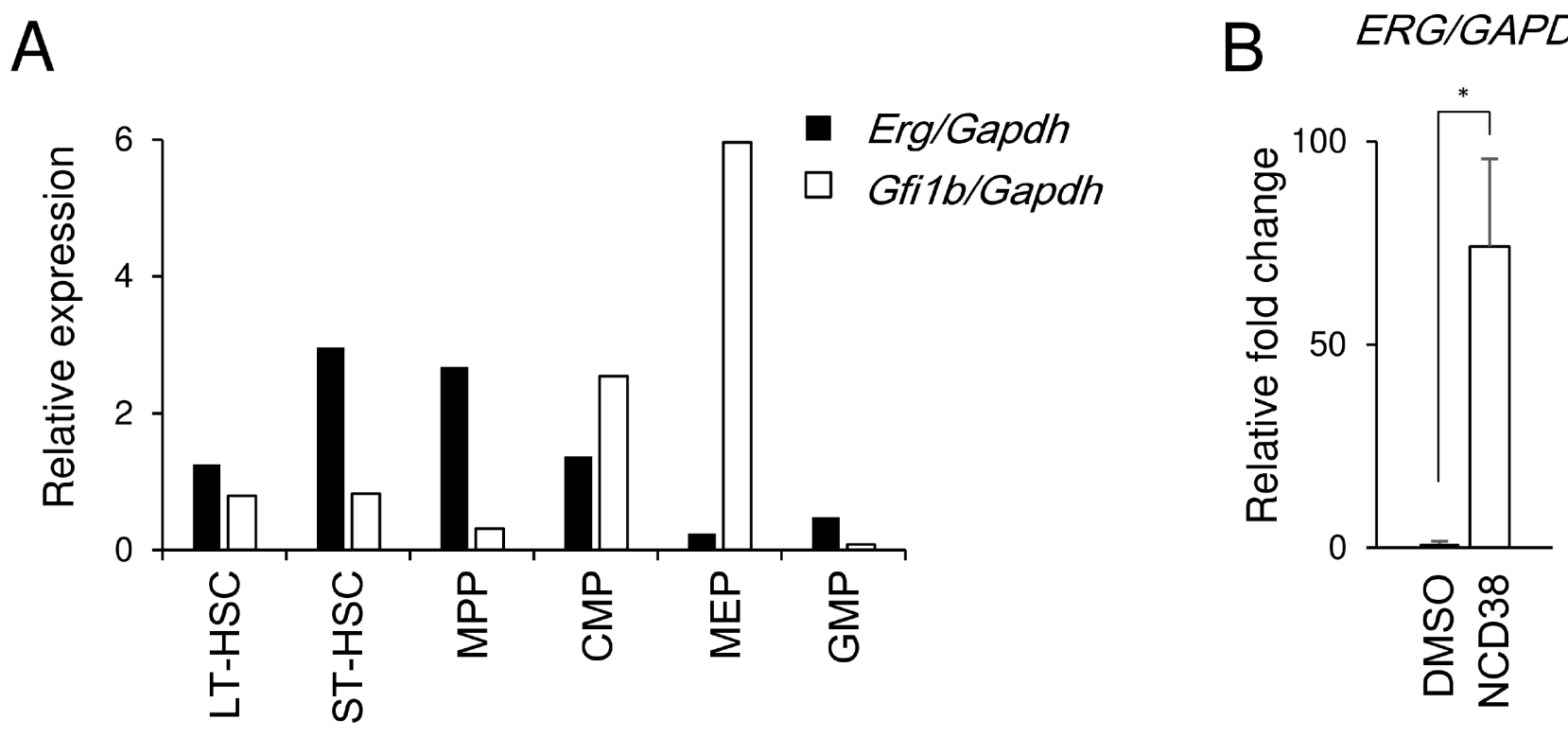

Figure 3: Inverse correlation between Erg and Gfi1b in MEP cells and de-repression of ERG by NCD38 in HEL cells. (A) Relative expression of the Erg and Gfilb transcripts of each hematopoietic fractions isolated from murine bone marrow. The data were normalized to the Gapdh transcript level. Experiments were performed independently twice and the means are displayed. LT-HSC, long-term hematopoietic stem cell; ST-HSC, short-term hematopoietic stem cell; MPP, multipotent progenitor; CMP, common myeloid progenitor; MEP, megakaryocyte-erythroid progenitor; GMP, granulocyte-monocyte progenitor. Sorting gates are shown in Supplementary Figure 1. (B) Relative fold change of the ERG transcript in HEL cells after treatment with NCD38 for 24 hours. The data are shown as the relative fold change in comparison to DMSO-treated HEL after normalization to GAPDH. The data are presented as mean with standard deviations for 3 independent experiments. Statistical comparison was performed using two-tailed Student $t$ test. ${ }^{*} P<0.01$. 
least in part, to anti-leukemia effects. It was an unexpected effect of NCD38 because NCD38 was originally designed to target the center of enzymatic activity and actually exhibits the strong inhibitory activity towards LSD1 [32]. However, the mechanism that NCD38 exerts antileukemia effects was unlikely to depend only on its inhibitory activity because NCD38 could quickly elevate the H3K27ac level on specific SEs despite the absence of DNA-binding motifs and deacetylation activity in LSD1 [15]. The current study could provide a new aspect of
NCD38 that makes it possible to explain these questions. Recently the specific protein-protein interactions (PPIs) have begun to be expected as novel drug targets [33]. The current findings suggest that the interaction between LSD1 and GFI1B is presumably an important PPI at least in erythroleukemia cells. In addition, it might be possible to classify NCD38 not only as a simple LSD1 inhibitor but also as a new PPI-targeting small molecule. According to the recent report, the interaction between LSD1 and GFI1B was also disrupted by another LSD1
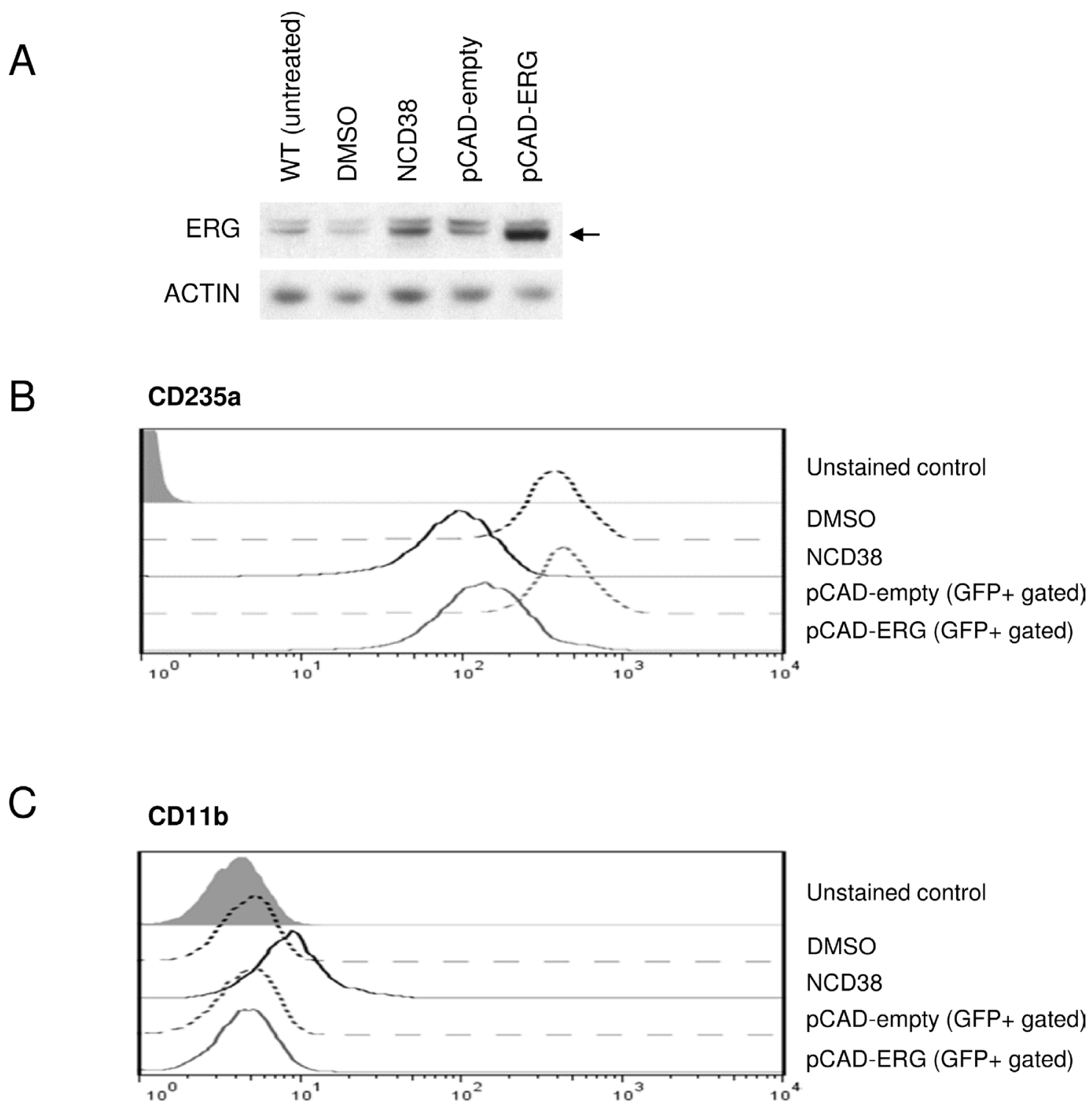

Figure 4: Lentiviral ERG overexpression mimics downregulation of the erythroid marker by NCD38. (A) ERG induction by NCD38 and overexpression by lentiviral transduction. Western blotting shows the ERG protein level (indicated by the arrow) in wildtype (WT, untreated), DMSO-treated, NCD38-treated, pCAD-empty-transduced, and pCAD-ERG-transduced HEL cells. Drug treatment time was 48 hours. ACTIN was used as an internal control. The schema of lentiviral vectors is shown in Supplementary Figure 2. (B-C) FACS analyses of CD235a (B) and CD11b (C). Histogram plots display CD235a or CD11b expression level on the cell surface of HEL cells treated with DMSO (black dotted line) or NCD38 (black solid line) for 48 hours, and of GFP-positive (GFP+ gated) HEL cells 3 days after transduction with pCAD-empty (gray dotted line) or pCAD-ERG (gray solid line). The gray filled histogram plots indicate unstained controls. The experiments were performed independently twice and the representative data are shown. 
inhibitor T-3775440 [18]. Although its selectivity of TFs and association with SEs were not mentioned, the basic structure of NCD38 and T-3775440 are very similar, implying that these drugs might act on leukemia cells through the same pharmacological mechanism. Structural analyses about how the GFI1B-LSD1 complex is altered and dissociated by these small molecules would be necessary to create more agile drugs which target the PPI between LSD1 and GFI1B.
GFI1B is highly expressed during erythroid and megakaryocytic maturation and suppresses nonerythroid specific genes [21]. Although several studies have reported that this suppression is regulated via the promoters $[34,35]$, our data propose that the repression of SEs by GFI1B and LSD1 may also play an important role in erythroid differentiation. The $E R G$-SE is identical to the $E R G$ stem cell enhancer in previous reports $[36,37]$. Several key hematopoietic TFs including
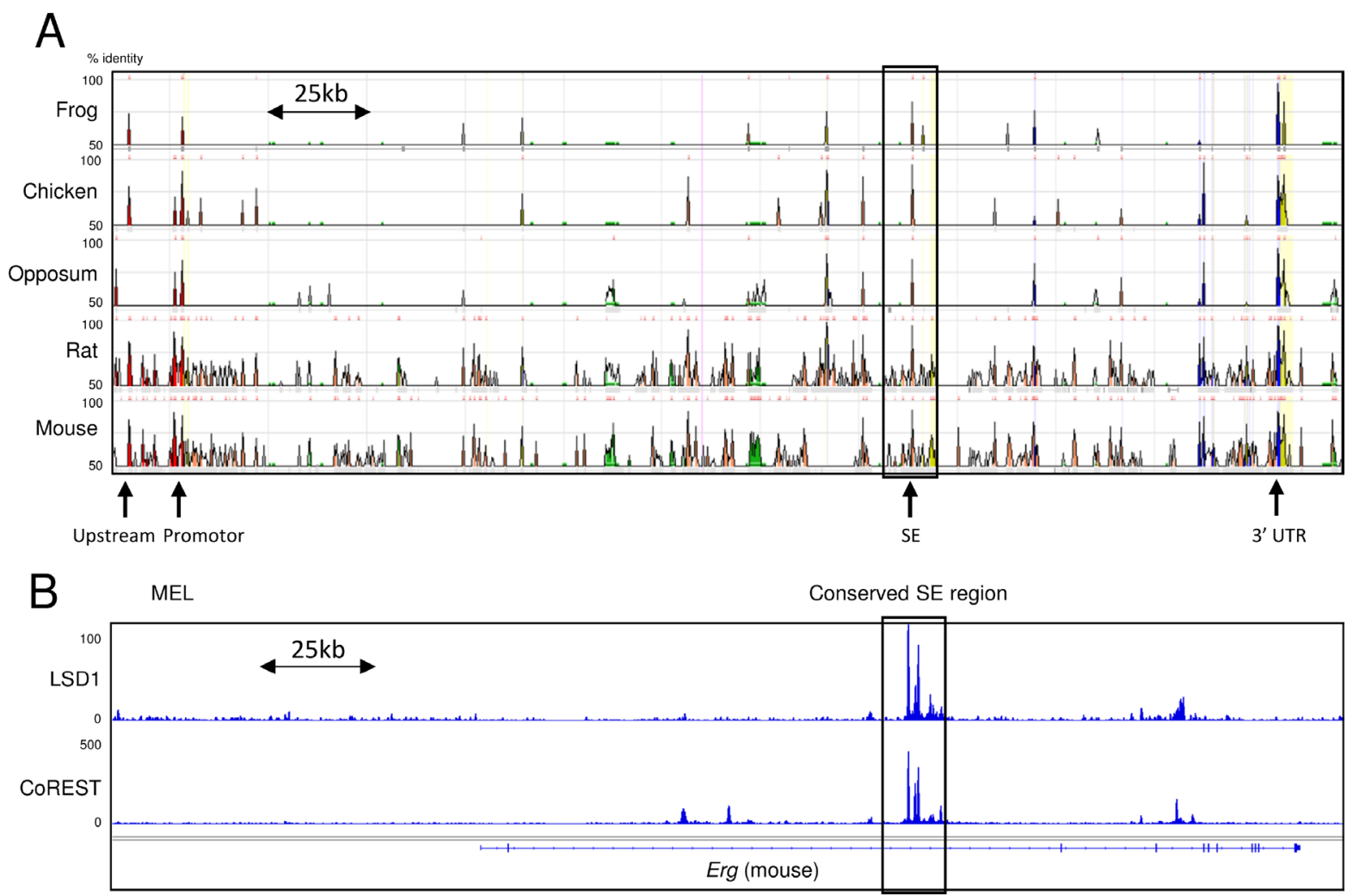

C

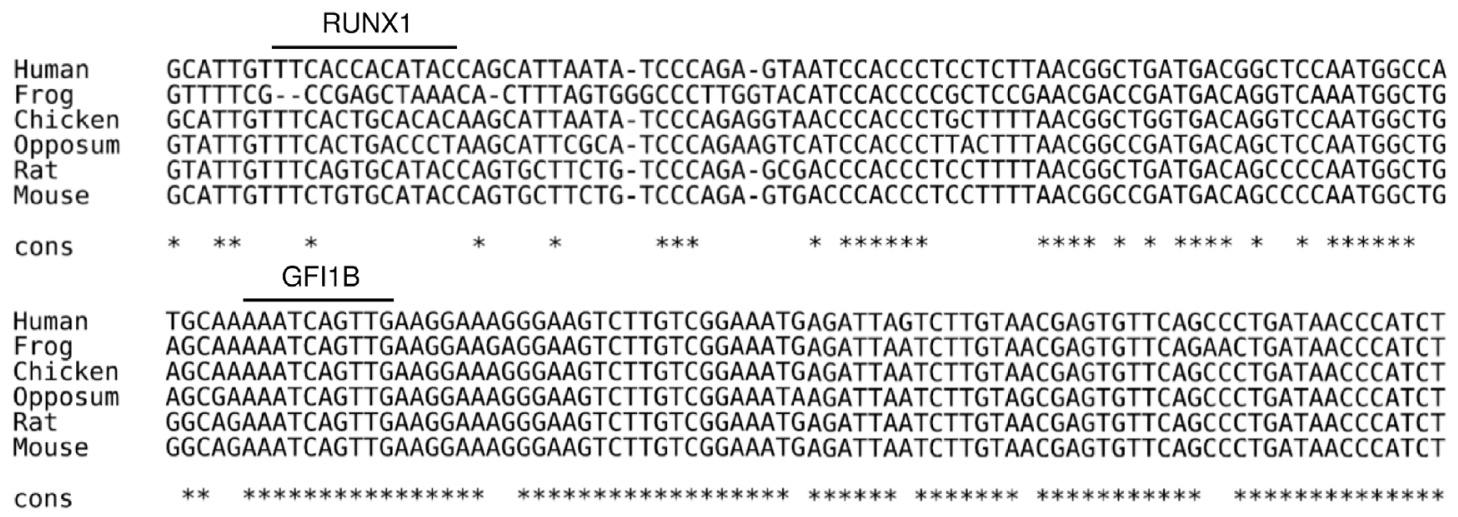

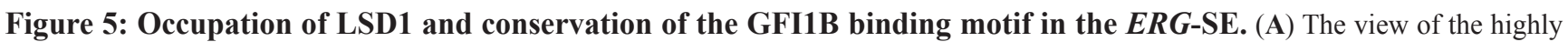
conserved regions at the ERG locus between human and the indicated species from ECR browser. The squared area is corresponding to the human $E R G$-SE. The height of the peaks indicates the degree of conservations between human and the indicated species. The arrows indicate highly conserved non-coding regions. (B) Publicly available ChIP-seq profiles of LSD1 and CoREST at the Erg locus in MEL cells. ChIP-seq data were visualized with IGV software. The squared area indicates the conserved SE region. (C) Alignment of the genomic sequences at the $E R G$-SE between the indicated species. The lines above the sequences indicate the binding motifs of RUNX1 or GFI1B. The asterisks indicate parts of the conserved sequences across the presented species. 
A

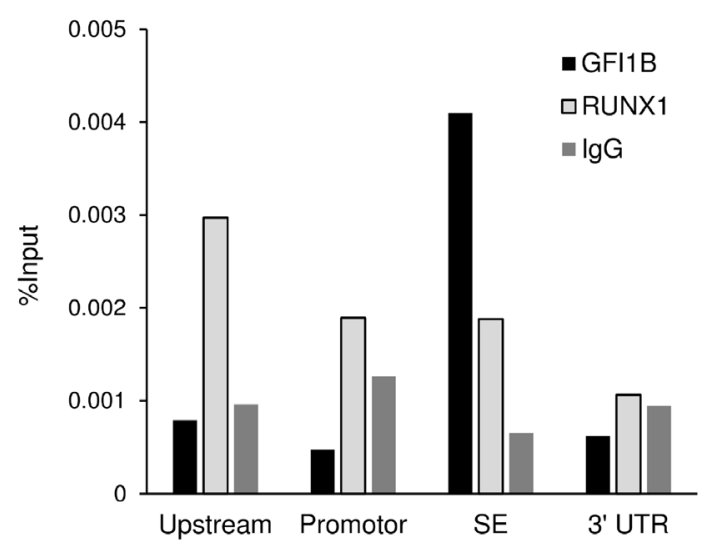

C

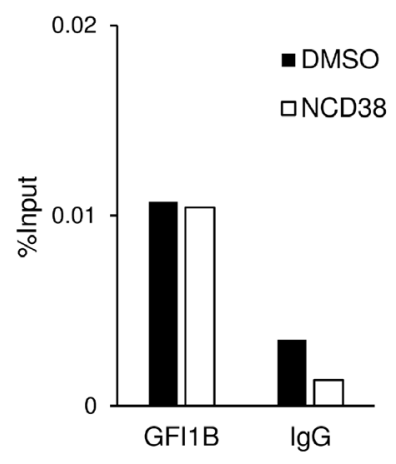

B

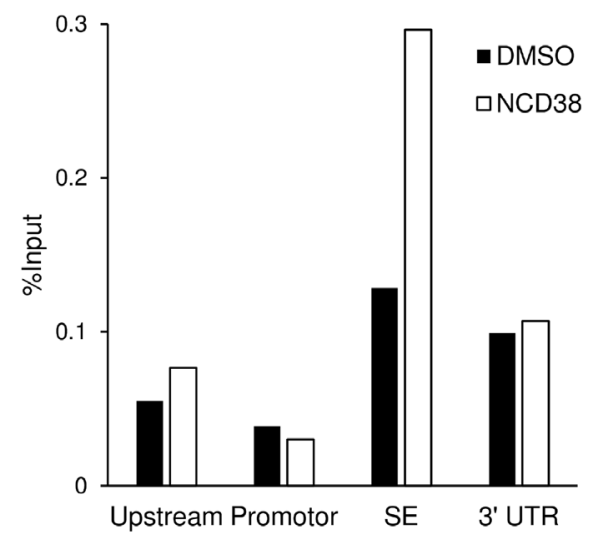

E

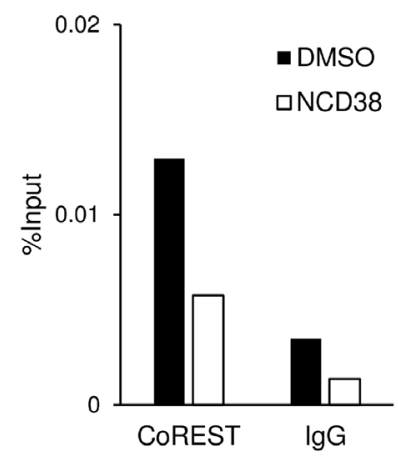

$\mathrm{F}$

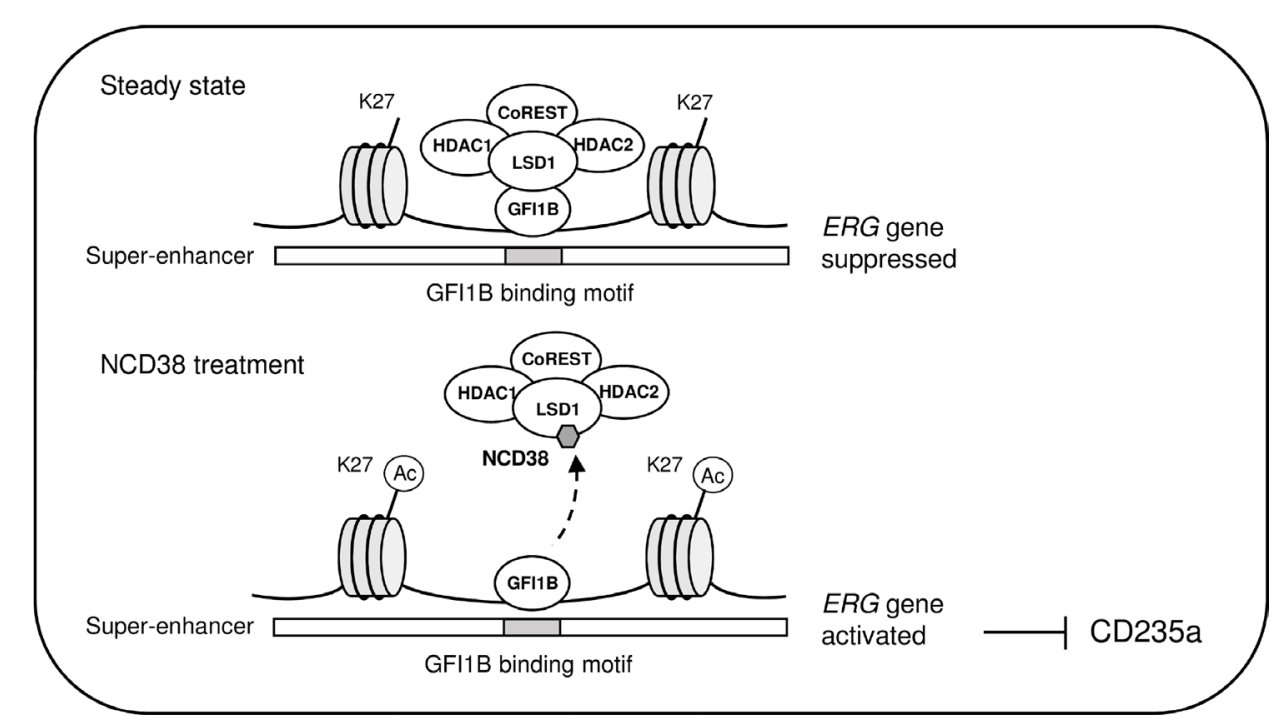

Figure 6: The $E R G$-SE is occupied by GFI1B and activated by the NCD38 treatment. (A) ChIP-qPCR analysis for GFI1B, RUNX1, or control IgG in the conserved non-coding regions at the $E R G$ locus in HEL cells. (B) ChIP-qPCR analysis for H3K27ac in the conserved non-coding regions at the $E R G$ locus in HEL cells treated with DMSO or NCD38 for 24 hours. Each conserved non-coding region is indicated in Figure 5A. (C-E) ChIP-qPCR analysis for GFI1B (C), LSD1 (D), and CoREST (E) at the ERG-SE locus in HEL cells treated with DMSO or NCD38 for 24 hours. All ChIP-qPCR assays were performed independently twice and the means are displayed. (F) Illustration of the interaction of GFI1B and the LSD1-CoREST-HDAC1/2 complex at the ERG-SE in HEL cells. In the steady state, GFI1B binds to the conserved binding motif and represses the $E R G$-SE through recruiting the LSD1-CoREST-HDAC1/2 complex. NCD38 separates the complex from GFI1B to restore the activity of the $E R G$-SE. 
SCL, LYL1, PU.1, LMO2, GATA2, RUNX1, FLI1 and ERG occupy and activate the ERG-SE leading to the upregulation of $E R G$ expression in human CD34 positive hematopoietic stem and progenitor cells. In addition, according to ChIP-seq analyses conducted in murine blood cells by another group, the H3K27ac level at the Erg-SE is the highest in MPPs but hardly detected in MEPs [6]. Therefore, downregulation of Erg in MEPs that was shown in Figure 3A might be caused by the repression of the ErgSE. On the other hand, it would be plausible to argue that this repression in MEPs might result from downregulation of PU.1 and Gata2 because they are known to be repressed in erythroid progenitors $[1,38]$. However, we previously reported that NCD38 could activate the ERG-SE without altering the transcript level of PU.1 and GATA2 in HEL cells [15]. The current study does not clarify whether Erg is downregulated especially in MEPs by exactly the same mechanism as shown in this report, but gives rise to the possibility that LSD1 and TFs may cooperate to silence lineage-specific regulators by inactivating their SEs and to control the cell fate. Further investigation what TFs are associated with LSD1 in each lineage will be required to better understand the precise hematopoietic transcriptional networks.

\section{MATERIALS AND METHODS}

\section{Cell culture and reagent}

HEL cells were maintained in RPMI-1640 supplemented with $10 \%$ heat-inactivated fetal bovine serum (FBS). Lenti-X 293T cells (Takara, Kusatsu, Japan) were grown in Dulbecco Modified Eagle's Medium (DMEM) supplemented with 10\% FBS. NCD38 was synthesized as previously described [32], dissolved in dimethyl sulfoxide (DMSO) and used at $2 \mu \mathrm{M}$ for all experiments.

\section{Mice}

C57BL/6 mice were purchased from Charles River Laboratories Japan (Yokohama, Japan) or CLEA Japan, Inc. (Tokyo, Japan), and maintained under specific pathogen-free conditions at the Centre for Experimental Animals of Kyoto University or at the Research Center for Animal Life Science of Shiga University of Medical Science. The animal experiments were approved by the animal research committee of Kyoto University or Shiga University of Medical Science and performed in accordance with the institutional guidelines.

\section{Antibodies}

The antibodies for immunoprecipitation were anti-LSD1 (ab17721, Abcam, Cambridge, UK), antiGFI1B (sc-28356X, Santa Cruz Biotech, Dallas, TX,
USA), anti-RUNX1 (ab23980, Abcam), anti-H3K27ac (39133, Active Motif, Carlsbad, CA, USA), anti-CoREST (ab32631, Abcam), normal rabbit IgG (sc-2027, Santa Cruz), and normal mouse IgG (sc-2025, Santa Cruz). The primary antibodies for western blotting were anti-LSD1 (C69G12, Millipore, Billerica, MA, USA), anti-CoREST (ab32631, Abcam), anti-HDAC1 (ab7028, Abcam), antiHDAC2 (ab7029, Abcam), anti-GFI1B (sc-28356X or sc22795, Santa Cruz), anti-RUNX1 (ab23980, Abcam, or sc-365644, Santa Cruz), anti-ERG (ab133264, Abcam), and anti-ACTIN (sc-1616, Santa Cruz). The secondary antibodies were anti-rabbit IgG (NA934V, GE Healthcare, Little Chalfont, UK), anti-mouse IgG (NA931V, GE Healthcare), and anti-goat IgG (sc-2020, Santa Cruz) conjugated with horseradish peroxidase.

\section{Co-immunoprecipitation assay}

For co-IP assays, $10^{7}$ cells were washed twice by PBS, lysed in $\mathrm{N} 450$ buffer $(50 \mathrm{mM}$ Tris- $\mathrm{HCl} \mathrm{pH} 7.5$, $450 \mathrm{mM} \mathrm{NaCl}, 1 \% \mathrm{NP}-40,5 \mathrm{mM}$ EDTA pH 8.0, $1 \mathrm{mM}$ $\mathrm{MgCl}_{2}, 5 \%$ Glycerol) and agitated for $30 \mathrm{~min}$ at $4^{\circ} \mathrm{C}$. After centrifugation, the supernatants were mixed with 2 volumes of N0 buffer (50 mM Tris-HCl pH 7.5, 1\% NP40, $5 \mathrm{mM}$ EDTA pH 8.0, $1 \mathrm{mM} \mathrm{MgCl}, 5 \%$ Glycerol). After preclear by incubation with $10 \mu \mathrm{L}$ of Dynabeads Protein A or G (Thermo Fisher Scientific, Waltham, MA, USA) for 30 min at $4^{\circ} \mathrm{C}$, the supernatants were incubated with $1-2 \mu \mathrm{g}$ of an indicated antibody for 1-3 hours at $4^{\circ} \mathrm{C}$, followed by immunoprecipitation with $20 \mu \mathrm{L}$ of the same beads for 3 hours at $4^{\circ} \mathrm{C}$. For co-IP with antiLSD1 antibody for mass spectrometry analyses or with anti-RUNX1 antibody, beads were conjugated with each antibody using the Dynabeads Antibody coupling kit (Thermo Fisher Scientific) according to the manufacturer's instruction and then used for 3-hour immunoprecipitation. The beads were washed five times with N150 buffer (50 $\mathrm{mM}$ Tris-HCl pH 7.5, $150 \mathrm{mM} \mathrm{NaCl}, 1 \% \mathrm{NP}-40,5 \mathrm{mM}$ EDTA pH 8.0, $1 \mathrm{mM} \mathrm{MgCl} 2,5 \%$ Glycerol) and then boiled for 5 min after adding sample buffer. For co-IP using antibody-conjugated beads, the beads were incubated with elution buffer $\left(0.5 \mathrm{M} \mathrm{NH}_{4} \mathrm{OH}, 0.5 \mathrm{mM}\right.$ EDTA pH $8.0)$ for $5 \mathrm{~min}$ at room temperature. The protease inhibitor cocktails (Nacalai Tesque, Kyoto, Japan) were added to N450, N0, and N150 buffers just before use. Silver staining was performed by using the Silver Staining MS kit (Wako, Osaka, Japan) according to the manufacturer's instruction.

\section{In-solution protein digestion}

The eluates from the immunoprecipitation beads were precipitated with cold acetone and resuspended in $8 \mathrm{M}$ Urea/30 $\mathrm{mM}$ ammonium bicarbonate. The resuspension was subjected to the reductive alkylation with dithiothreitol and iodoacetamide. One $\mu \mathrm{g}$ of tosyl 
phenylalanyl chloromethyl ketone-treated trypsin (Thermo Fisher Scientific) was added to the solution and the proteins were digested for overnight. The digestion products were purified using C-18 spin column (Thermo Fisher Scientific) according to the manufacturers' instruction, and resuspended in $0.1 \%$ formic acid before subjection to the mass spectrometry.

\section{Mass spectrometry}

The protein digests were separated using Nano-LCUltra 2D-plus equipped with cHiPLC Nanoflex (Eksigent, Dublin, CA, USA) in trap-and-elute mode, with trap column $(200 \mu \mathrm{m} \times 0.5 \mathrm{~mm}$ ChromXP C18-CL $3 \mu \mathrm{m} 120$ $\AA$ (Eksigent) $)$ and analytical column $(75 \mu \mathrm{m} \times 15 \mathrm{~cm}$ ChromXP C18-CL $3 \mu \mathrm{m} 120 \AA$ (Eksigent)). The separation was carried out with a binary gradient with solvent A ( $98 \%$ water, $2 \%$ acetonitrile, $0.1 \%$ formic acid) and solvent $\mathrm{B}$ ( $20 \%$ water, $80 \%$ acetonitrile, $0.1 \%$ formic acid). The gradient program was 2 to $40 \%$ B for 250 min, 40 to $90 \%$ B in $1 \mathrm{~min}, 90 \% \mathrm{~B}$ for $5 \mathrm{~min}, 90$ to $2 \% \mathrm{~B}$ in $0.1 \mathrm{~min}$, and $2 \%$ $B$ for $18.9 \mathrm{~min}$, at $300 \mathrm{~nL} / \mathrm{min}$. The eluates were infused on-line to a mass spectrometer (TripleTOF $5600+$ System with NanoSpray III source and heated interface (SCIEX, Framingham, MA, USA)) and ionized in an electrospray ionization-positive mode. Data acquisition was carried out with an information-dependent acquisition method. The acquired datasets were analyzed by ProteinPilot software version 4.5beta (SCIEX) with the UniProtKB/Swiss-Prot database for human (June 2014) appended with known common contaminants (SCIEX). The quality of the database search was confirmed by the false discovery rate analysis in which the reversed amino acid sequences were used as decoy. The protein identifications were evaluated by the numbers of identified peptides with at least $95 \%$ confidence, and the corresponding Unused ProtScores that were calculated by the Pro Group algorithm (SCIEX).

\section{Label-free quantification of the relative protein abundance}

The LC-MS/MS datasets acquired by TripleTOF 5600 + System were imported to the platform of Progenesis QI for proteomics software (Nonlinear Dynamics, Newcastle upon Tyne, UK) for the relative quantification [39]. The relative abundance of each peptide was calculated using normalize to all proteins method. The identification of peptides was carried out by importing the corresponding peptide identification results generated by ProteinPilot software. The relative abundance of each protein was calculated by the grouping non-conflicting peptides method.

\section{Real-time quantitative PCR}

RNA extractions were prepared using TRIzol reagent (Thermo Fisher Scientific) or the RNeasy
Micro Kit (Qiagen, Hilden, Germany), and cDNAs were synthesized by Superscript II reverse transcriptase and Oligo (dT) primers (Thermo Fisher Scientific). Quantitative PCR (qPCR) of mRNA and ChIP-DNA were performed using Thunderbird SYBR qPCR Mix (Toyobo, Osaka, Japan) and TaKaRa Dice Real-Time TP800 system (Takara), or LightCycler 2.0 instrument (Roche Applied Science, Mannheim, Germany). Primer sequences are shown in Supplementary Table 2.

\section{Flow cytometry}

For sorting murine hematopoietic stem and progenitor cells, FITC-conjugated anti-CD34 (RAM34), PE-conjugated anti-FcgRII/III (93), pacific blueconjugated anti-Scal (D7), APC-conjugated anti-cKit (2B8), and biotin-conjugated anti-CD135 (A2F10) antibodies followed by APC-Cy7-conjugated streptavidin were used. For excluding lineage positive cells, PECy5-conjugated anti-CD3e (145-2C11), CD4 (GK1.5), CD8a (53-6.7), CD19 (eBio1D3), B220 (RA3-6B2), Gr-1 (RB6-8C5), TER-119 (TER-119), and CD11b (M1/70) antibodies were used. Cell sorting experiments were performed using a FACS Aria flow cytometer (BD Biosciences, San Jose, CA, USA). For flow cytometric analysis of HEL cells, PE-Cy5-conjugated anti-human CD11b (ICRF44) and CD235a (GA-R2) antibodies were used. Flow cytometric analyses were performed using a FACS Calibur (BD Biosciences). These antibodies were purchased from eBioscience (San Diego, CA, USA), BD Biosciences, or Biolegend (San Diego, CA, USA).

\section{Chromatin immunoprecipitation (ChIP)}

ChIP assays for GFI1B, RUNX1, H3K27ac, LSD1 and CoREST were performed as described previously [15]. Briefly, after crosslinking with $0.5 \%$ or $1 \%$ paraformaldehyde for $10 \mathrm{~min}$ and quenching with 100 $\mathrm{mM}$ glycine for $10 \mathrm{~min}$, cells were lysed and incubated in lysis buffer (50 mM HEPES pH 7.9, $140 \mathrm{mM} \mathrm{NaCl}, 1$ mM EDTA pH 8.0, 10\% Glycerol, 0.5\% NP-40, 0.25\% TritonX-100) on ice for $10 \mathrm{~min}$. After two washes, the pellets were resuspended in shearing buffer $(0.1 \%$ SDS, 1 mM EDTA pH 8.0, $10 \mathrm{mM}$ Tris-HCl pH 8.0) and sonicated using S220 ultrasonicators (Covaris, Woburn, MA, USA). The sonicated samples were incubated overnight at $4^{\circ} \mathrm{C}$ with 2 or $4 \mu \mathrm{g}$ of an indicated antibody and precipitated with $20 \mu \mathrm{L}$ of Dynabeads Protein A or protein G. After extensive wash, the precipitated magnetic beads were treated with elution buffer $(50 \mathrm{mM}$ Tris- $\mathrm{HCl} \mathrm{pH} 8.0,10$ mM EDTA pH 8.0, 1\% SDS). Cross-links of the eluted samples were reversed by incubation for overnight at $65^{\circ} \mathrm{C}$. After digestion of RNA and protein with RNase A and Proteinase K, DNA was finally purified using a Min Elute PCR Purification Kit (Qiagen) and analyzed by realtime qPCR. 


\section{Lentiviral transduction}

For $E R G$ overexpression, the pCMV-Sport6 vector encoding the full-sequence human $E R G$ gene (Clone ID: 6052140, GenBank ${ }^{\mathrm{TM}}$ accession number: BC040168) was purchased from Dharmacon (Lafayette, CO, USA) and the $E R G$ coding part was moved to pCAD lentiviral vector equipped with IRES-GFP [4]. Lentiviral supernatants were generated in Lenti-X 293T cells as previously described [4], and infected to HEL cells for 24 hours. After 3 days, transduced HEL cells were analyzed by FACS. After 5 days, GFP positive cells were sorted using a FACS Aria flow cytometer (BD Biosciences), lysed in RIPA buffer (10 mM Tris-HCl pH 7.5, $150 \mathrm{mM} \mathrm{NaCl}, 5 \mathrm{mM}$ EDTA pH $8.0,1 \%$ Triton X-100, $1 \%$ sodium deoxycholate, $0.1 \%$ SDS, $1 \mathrm{mM}$ PMSF) with the protease inhibitor cocktail, and analyzed by western blotting.

\section{ChIP-seq analysis, identification of conserved genomic regions, and TF motif analysis}

The publicly available ChIP-seq data obtained from Gene Expression Omnibus database; LSD1 (Series GSE59859; Sample GSM1448833) [24], and CoREST (Series GSE36030; Sample GSM1003789) [25, 26] were visualized with the Integrative Genomics Viewer (IGV) software version 2.3 (Broad Institute, Cambridge, MA, USA; https://www.broadinstitute.org/igv). Genomic regions conserved among the species were identified using ECR browser (http://ecrbrowser.dcode.org/). Genomic sequence spanning the $E R G$ locus was obtained from UCSC genome browser (https://genome.ucsc.edu/). DNA sequence alignments were performed using T-coffee server (http://tcoffee.vital-it.ch/apps/tcoffee/do:regular). TF binding sites were analyzed with R-VISTA (http://rvista. dcode.org/).

\section{Statistics}

Statistical comparison was performed using twotailed Student $t$ test.

\section{Author contributions}

The study was designed and conducted by MK and supervised by MH, TS, and AA. The study was performed by RY, GT, SI, JS, and MK. The data was analyzed by RY, SI and MK. The manuscript was composed by RY, SI and MK. The compound was synthesized and provided by TS. All authors read and approved of the manuscript.

\section{ACKNOWLEDGMENTS}

Mass spectrometry and sonication for ChIP assay were performed at the Medical Research Support Center, Graduate School of Medicine, Kyoto University, which was supported by Platform for Drug Discovery,
Informatics, and Structural Life Science from the Ministry of Education, Culture, Sports, Science and Technology, Japan. We thank Dr. Akifumi Takaori (Kyoto University) for some support for performing experiments.

\section{CONFLICTS OF INTEREST}

The authors have declared that they have no competing interests.

\section{FUNDING}

This work was supported by the JSPS KAKENHI Grant Number JP16K09846 (MK) and the Takeda Science Foundation (MK).

\section{REFERENCES}

1. Tenen DG. Disruption of differentiation in human cancer: AML shows the way. Nat Rev Cancer. 2003; 3:89-101.

2. Patel JP, Gonen M, Figueroa ME, Fernandez H, Sun Z, Racevskis J, Van Vlierberghe P, Dolgalev I, Thomas $\mathrm{S}$, Aminova O, Huberman $\mathrm{K}$, Cheng J, Viale A, et al. Prognostic relevance of integrated genetic profiling in acute myeloid leukemia. N Engl J Med. 2012; 366:1079-1089.

3. Rosenbauer F, Wagner K, Kutok JL, Iwasaki H, Le Beau MM, Okuno Y, Akashi K, Fiering S, Tenen DG. Acute myeloid leukemia induced by graded reduction of a lineage-specific transcription factor, PU.1. Nat Genet. 2004; 36:624-630.

4. Kawahara M, Pandolfi A, Bartholdy B, Barreyro L, Will B, Roth M, Okoye-Okafor UC, Todorova TI, Figueroa ME, Melnick A, Mitsiades CS, Steidl U. H2.0-like homeobox regulates early hematopoiesis and promotes acute myeloid leukemia. Cancer Cell. 2012; 22:194-208.

5. Moignard V, Macaulay IC, Swiers G, Buettner F, Schutte J, Calero-Nieto FJ, Kinston S, Joshi A, Hannah R, Theis FJ, Jacobsen SE, de Bruijn MF, Gottgens B. Characterization of transcriptional networks in blood stem and progenitor cells using high-throughput single-cell gene expression analysis. Nat Cell Biol. 2013; 15:363-372.

6. Lara-Astiaso D, Weiner A, Lorenzo-Vivas E, Zaretsky I, Jaitin DA, David E, Keren-Shaul H, Mildner A, Winter D, Jung S, Friedman N, Amit I. Immunogenetics. Chromatin state dynamics during blood formation. Science. 2014; 345:943-949.

7. Whyte WA, Orlando DA, Hnisz D, Abraham BJ, Lin CY, Kagey MH, Rahl PB, Lee TI, Young RA. Master transcription factors and mediator establish super-enhancers at key cell identity genes. Cell. 2013; 153:307-319.

8. Hnisz D, Abraham BJ, Lee TI, Lau A, Saint-Andre V, Sigova AA, Hoke HA, Young RA. Super-enhancers in the control of cell identity and disease. Cell. 2013; 155:934-947.

9. Shi Y, Lan F, Matson C, Mulligan P, Whetstine JR, Cole PA, Casero RA, Shi Y. Histone demethylation mediated 
by the nuclear amine oxidase homolog LSD1. Cell. 2004; 119:941-953.

10. Shi YJ, Matson C, Lan F, Iwase S, Baba T, Shi Y. Regulation of LSD1 histone demethylase activity by its associated factors. Mol Cell. 2005; 19:857-864.

11. Lee MG, Wynder C, Cooch N, Shiekhattar R. An essential role for CoREST in nucleosomal histone 3 lysine 4 demethylation. Nature. 2005; 437:432-435.

12. Shlyueva D, Stampfel G, Stark A. Transcriptional enhancers: from properties to genome-wide predictions. Nat Rev Genet. 2014; 15:272-286.

13. Heintzman ND, Stuart RK, Hon G, Fu Y, Ching CW, Hawkins RD, Barrera LO, Van Calcar S, Qu C, Ching KA, Wang W, Weng Z, Green RD, et al. Distinct and predictive chromatin signatures of transcriptional promoters and enhancers in the human genome. Nat Genet. 2007; 39:311-318.

14. Kerenyi MA, Shao Z, Hsu YJ, Guo G, Luc S, O’Brien K, Fujiwara Y, Peng C, Nguyen M, Orkin SH. Histone demethylase Lsd1 represses hematopoietic stem and progenitor cell signatures during blood cell maturation. eLife. 2013; 2:e0633.

15. Sugino N, Kawahara M, Tatsumi G, Kanai A, Matsui H, Yamamoto R, Nagai Y, Fujii S, Shimazu Y, Hishizawa M, Inaba T, Andoh A, Suzuki T, Takaori-Kondo A. A novel LSD1 inhibitor NCD38 ameliorates MDS-related leukemia with complex karyotype by attenuating leukemia programs via activating super-enhancers. Leukemia. 2017; 31:2303-2314.

16. Hakimi MA, Bochar DA, Chenoweth J, Lane WS, Mandel G, Shiekhattar R. A core-BRAF35 complex containing histone deacetylase mediates repression of neuronal-specific genes. Proc Natl Acad Sci U S A. 2002; 99:7420-7425.

17. Saleque S, Kim J, Rooke HM, Orkin SH. Epigenetic regulation of hematopoietic differentiation by Gfi-1 and Gfi- $1 \mathrm{~b}$ is mediated by the cofactors CoREST and LSD1. Mol Cell. 2007; 27:562-572.

18. Ishikawa Y, Gamo K, Yabuki M, Takagi S, Toyoshima K, Nakayama K, Nakayama A, Morimoto M, Miyashita H, Dairiki R, Hikichi Y, Tomita N, Tomita D, et al. A Novel LSD1 Inhibitor T-3775440 Disrupts GFI1B-Containing Complex Leading to Transdifferentiation and Impaired Growth of AML Cells. Mol Cancer Ther. 2017; 16:273-284.

19. Loughran SJ, Kruse EA, Hacking DF, de Graaf CA, Hyland CD, Willson TA, Henley KJ, Ellis S, Voss AK, Metcalf D, Hilton DJ, Alexander WS, Kile BT. The transcription factor Erg is essential for definitive hematopoiesis and the function of adult hematopoietic stem cells. Nat Immunol. 2008; 9:810-819.

20. Taoudi S, Bee T, Hilton A, Knezevic K, Scott J, Willson TA, Collin C, Thomas T, Voss AK, Kile BT, Alexander WS, Pimanda JE, Hilton DJ. ERG dependence distinguishes developmental control of hematopoietic stem cell maintenance from hematopoietic specification. Genes Dev. 2011; 25:251-262.

21. Moroy T, Vassen L, Wilkes B, Khandanpour C. From cytopenia to leukemia: the role of Gfil and Gfilb in blood formation. Blood. 2015; 126:2561-2569.

22. Pronk CJ, Rossi DJ, Mansson R, Attema JL, Norddahl GL, Chan CK, Sigvardsson M, Weissman IL, Bryder D. Elucidation of the phenotypic, functional, and molecular topography of a myeloerythroid progenitor cell hierarchy. Cell Stem Cell. 2007; 1:428-442.

23. Saleque S, Cameron S, Orkin SH. The zinc-finger protooncogene Gfi-1b is essential for development of the erythroid and megakaryocytic lineages. Genes Dev. 2002; 16:301-306.

24. Stadhouders R, Cico A, Stephen T, Thongjuea S, Kolovos P, Baymaz HI, Yu X, Demmers J, Bezstarosti K, Maas A, Barroca V, Kockx C, Ozgur Z, et al. Control of developmentally primed erythroid genes by combinatorial co-repressor actions. Nat Commun. 2015; 6:8893.

25. Soler E, Andrieu-Soler C, de Boer E, Bryne JC, Thongjuea S, Stadhouders R, Palstra RJ, Stevens M, Kockx C, van Ijcken W, Hou J, Steinhoff C, Rijkers E, et al. The genomewide dynamics of the binding of Ldb1 complexes during erythroid differentiation. Genes Dev. 2010; 24:277-289.

26. Ram O, Goren A, Amit I, Shoresh N, Yosef N, Ernst J, Kellis M, Gymrek M, Issner R, Coyne M, Durham T, Zhang $\mathrm{X}$, Donaghey J, et al. Combinatorial patterning of chromatin regulators uncovered by genome-wide location analysis in human cells. Cell. 2011; 147:1628-1639.

27. van Riel B, Pakozdi T, Brouwer R, Monteiro R, Tuladhar K, Franke V, Bryne JC, Jorna R, Rijkers EJ, van Ijcken W, Andrieu-Soler C, Demmers J, Patient R, et al. A novel complex, RUNX1-MYEF2, represses hematopoietic genes in erythroid cells. Mol Cell Biol. 2012; 32:3814-3822.

28. Hu X, Li X, Valverde K, Fu X, Noguchi C, Qiu Y, Huang $\mathrm{S}$. LSD1-mediated epigenetic modification is required for TAL1 function and hematopoiesis. Proc Natl Acad Sci U S A. 2009; 106:10141-10146.

29. Porcher C, Swat W, Rockwell K, Fujiwara Y, Alt FW, Orkin $\mathrm{SH}$. The T cell leukemia oncoprotein SCL/tal-1 is essential for development of all hematopoietic lineages. Cell. 1996; 86:47-57.

30. Schenk T, Chen WC, Gollner S, Howell L, Jin L, Hebestreit K, Klein HU, Popescu AC, Burnett A, Mills K, Casero RA Jr, Marton L, Woster P, et al. Inhibition of the LSD1 (KDM1A) demethylase reactivates the all-trans-retinoic acid differentiation pathway in acute myeloid leukemia. Nat Med. 2012; 18:605-611.

31. Harris WJ, Huang X, Lynch JT, Spencer GJ, Hitchin JR, Li Y, Ciceri F, Blaser JG, Greystoke BF, Jordan AM, Miller CJ, Ogilvie DJ, Somervaille TC. The histone demethylase KDM1A sustains the oncogenic potential of MLL-AF9 leukemia stem cells. Cancer Cell. 2012; 21:473-487. 
32. Ogasawara D, Itoh $\mathrm{Y}$, Tsumoto $\mathrm{H}$, Kakizawa $\mathrm{T}$, Mino K, Fukuhara K, Nakagawa H, Hasegawa M, Sasaki R, Mizukami T, Miyata N, Suzuki T. Lysine-specific demethylase 1-selective inactivators: protein-targeted drug delivery mechanism. Angew Chem Int Ed Engl. 2013; 52:8620-8624.

33. Scott DE, Bayly AR, Abell C, Skidmore J. Small molecules, big targets: drug discovery faces the proteinprotein interaction challenge. Nat Rev Drug Discov. 2016; 15:533-550.

34. Vassen L, Fiolka K, Moroy T. Gfilb alters histone methylation at target gene promoters and sites of gammasatellite containing heterochromatin. EMBO J. 2006; 25:2409-2419.

35. Kuo YY, Chang ZF. GATA-1 and Gfi-1B interplay to regulate Bcl-xL transcription. Mol Cell Biol. 2007; $27: 4261-4272$.

36. Thoms JA, Birger Y, Foster S, Knezevic K, Kirschenbaum $\mathrm{Y}$, Chandrakanthan V, Jonquieres G, Spensberger D,
Wong JW, Oram SH, Kinston SJ, Groner Y, Lock R, et al. ERG promotes T-acute lymphoblastic leukemia and is transcriptionally regulated in leukemic cells by a stem cell enhancer. Blood. 2011; 117:7079-7089.

37. Diffner E, Beck D, Gudgin E, Thoms JA, Knezevic K, Pridans C, Foster S, Goode D, Lim WK, Boelen L, Metzeler KH, Micklem G, Bohlander SK, et al. Activity of a heptad of transcription factors is associated with stem cell programs and clinical outcome in acute myeloid leukemia. Blood. 2013; 121:2289-2300.

38. Briegel K, Bartunek P, Stengl G, Lim KC, Beug H, Engel JD, Zenke M. Regulation and function of transcription factor GATA-1 during red blood cell differentiation. Development. 1996; 122:3839-3850.

39. Silva JC, Gorenstein MV, Li GZ, Vissers JP, Geromanos SJ. Absolute quantification of proteins by LCMSE: a virtue of parallel MS acquisition. Mol Cell Proteomics. 2006; $5: 144-156$. 